\title{
Limulus amoebocyte lysate assay of endotoxin: a method for visual detection of the positive gel reaction
}

\author{
S. N. HUSSAINI and H.T. HASSANALI
}

Ministry of Agriculture, Fisheries and Food, Central Veterinary Laboratory, Weybridge, Surrey, KT15 3NB

\begin{abstract}
Summary. In a Limulus amoebocyte lysate micro-test system, visual differentiation between positive and negative reactions was obtained by the addition of a small drop of fresh, defibrinated sheep blood to the reaction mixture. This method has greatly facilitated end-point identification in a series of dilutions of endotoxin.
\end{abstract}

The Limulus amoebocyte lysate (LAL) test is currently employed to detect and quantitate endotoxins in various biological products for parenteral administration, e.g., vaccines, sera, plasma (Kreeftenberg et al., 1977; Rastogi et al., 1977; Hussaini and Ready, 1981; Mortensen and Binder, 1985). The test is being used with growing frequency in veterinary and human medicine in Europe and America. In the USA it has replaced the costly invivo test in rabbits as the mandatory test for pyrogen testing of water for injection and of a range of radiopharmaceuticals (Wiblin, 1985).

The LAL test is very simple to perform. In the micro-test system $10-\mu \mathrm{l}$ volumes (drops) of test material are mixed with the lysate in a sterile petri dish and incubated undisturbed at $37^{\circ} \mathrm{C}$ for $30 \mathrm{~min}$. A positive reaction is characterised by gel formation, whereas in a negative reaction the mixture remains liquid. The distinction is made either by tilting the plate to an angle or by gently tapping the side of the plate; the positive gel remains immovable whereas the negative drop flows or quivers. Visualisation of the reaction is improved when a small amount $(<5 \mu \mathrm{l})$ of methylene blue $0 \cdot 1 \%$ is added (Hussaini and Sawtell, 1986) but the problem of subjectivity in interpreting end points of less than complete gelation is not resolved by this method.

As an alternative to the cumbersome enzymic method which measures cleavage of a synthetic chromogenic substrate by the clottable enzyme (Harada et al., 1979), we report here a simple and rapid method for the visual confirmation of a positive reaction by use of fresh, defibrinated sheep blood. In a micro-test application, as described earlier, in which the reaction mixtures are discrete

Received 14 Nov. 1986; revised version accepted 14 Jan. 1987. drops, gelation is tested by adding a small drop ( $<5 \mu \mathrm{l}$ to $20 \mu \mathrm{l}$ of the reaction mixture) of fresh defibrinated sheep blood, by means of a microsyringe, on to the surface of the reaction mixture. If gelation has occurred (positive reaction) the red blood cells remain held together within the drop in the shape of a bright red button surrounded by a clear zone (figure, drop 1). By contrast, in a negative reaction (no gelation) the blood immediately disperses homogeneously throughout the entire drop (figure, drop 4). In a series of dilutions, as the concentration of endotoxin decreases the size of the

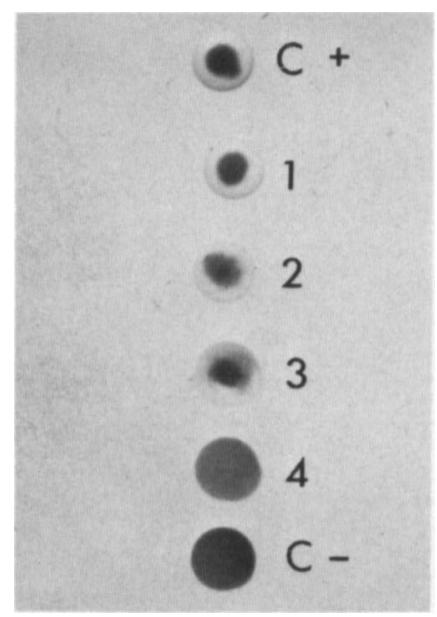

Figure. Test mixture drops in a sterile petri dish. Lyophilised 'Pyrogent' reagent (Mallinckrodt) with a labelled sensitivity of $0.012 \mathrm{ng}$ (0.06 Endotoxin Units) of Escherichia coli endotoxin per $\mathrm{ml}$ was used. This was reconstituted in pyrogen-free water and $10-\mu l$ volumes were added to $10-\mu l$ samples containing $E$. coli endotoxin ( $E$. coli vaccine) and a control. The concentrations of endotoxin in drops $1-4$ were $1 \cdot 2,0.12,0.012$ and $0 \mathrm{ng} / \mathrm{ml}$ respectively. The results were read by placing $5 \mu \mathrm{l}$ of fresh, defibrinated sheep blood on top of each drop. $\mathrm{C}+$, positive control (lysate $+E$. coli endotoxin); $\mathrm{C}-$, negative control (lysate + pyrogen-free water). 
button increases, with easily perceived reduction in the width of the clear zone surrounding it. This makes the determination of the end point easier than with conventional observation (figure, drops 2 and 3). Other types of blood, such as commercial or fresh defibrinated horse or chicken blood have been tried but do not give such clear results as sheep blood.

We have used this rapid identification procedure to quantify endotoxins in gram-negative bacterial vaccines and sera and have found fresh defibrinated sheep blood to contribute substantially as a visual

\section{REFERENCES}

Harada T, Morita T, Iwanaga S, Nakamura S, Niwa M 1979 A new chromogenic substrate method for assay of bacterial endotoxins using Limulus hemocyte lysate. In: Cohen E (ed) Biomedical application of the horseshoe crab (Limulidae), Progress in Clinical and Biological Research 29, Alan R Liss, Inc., New York, pp 209-220.

Hussaini S N, Ready R A 1981 Studies on Escherichia coli vaccines: estimation of endotoxins in veterinary vaccines. Veterinary Research Communications 5: 171-175.

Hussaini S N, Sawtell J A A 1986 Studies on Escherichia coli vaccines: an in vitro approach to establishing safe limits of endotoxins in vaccines. Developments in Biological Standardization 64: 261-269. aid in confirming borderline positive results otherwise misinterpreted as negative without the aid of the blood. In a borderline positive gelation result, the central button is surrounded by a diffuse zone of red blood cells (figure, drop 3). The dynamics of the process by which the red blood cells do not disperse, however, have not been examined. We presume that the gel matrix, in a positive reaction, prevents the dispersal of the red blood cells thus rounding them into a spot, whereas the liquid state of a negative reaction allows them to disperse evenly within the drop.

Kreeftenberg J G, Loggen H G, van Ramhorst J D, Beuvery E C 1977 The Limulus amoebocyte lysate test micromethod and application in the control of sera and vaccines. Developments in Biological Standardization 34: 15-20.

Mortensen K, Binder M 1985 The Limulus amoebocyte lysate test: Detection of endotoxin in plasma of swine and cattle. 1. In vitro investigations. Acta Veterinaria Scandinavica 26 : 231-245.

Rastogi S C, Hochstein H D, Seligmann E B 1977 Statistical determination of endotoxin content in influenza virus vaccine by the Limulus amoebocyte lysate test. Journal of Clinical Microbiology 6: 144-148.

Wiblin C N 1985 Pyrogenicity and carcinogenicity tests. In: Speir R E, Griffiths J B (eds) Animal cell biotechnology, vol 2, Academic Press, London, pp 321-354. 